\title{
Review Article \\ Effects of Caloric Restriction on Cardiac Oxidative Stress and Mitochondrial Bioenergetics: Potential Role of Cardiac Sirtuins
}

\author{
Ken Shinmura \\ Division of Geriatric Medicine, Department of Internal Medicine, Keio University School of Medicine, 35 Shinanomachi, \\ Shinjuku-ku, Tokyo 160-8582, Japan \\ Correspondence should be addressed to Ken Shinmura; shimmura@z5.keio.jp
}

Received 10 February 2013; Accepted 18 February 2013

Academic Editor: Nilanjana Maulik

Copyright (C) 2013 Ken Shinmura. This is an open access article distributed under the Creative Commons Attribution License, which permits unrestricted use, distribution, and reproduction in any medium, provided the original work is properly cited.

The biology of aging has not been fully clarified, but the free radical theory of aging is one of the strongest aging theories proposed to date. The free radical theory has been expanded to the oxidative stress theory, in which mitochondria play a central role in the development of the aging process because of their critical roles in bioenergetics, oxidant production, and regulation of cell death. A decline in cardiac mitochondrial function associated with the accumulation of oxidative damage might be responsible, at least in part, for the decline in cardiac performance with age. In contrast, lifelong caloric restriction can attenuate functional decline with age, delay the onset of morbidity, and extend lifespan in various species. The effect of caloric restriction appears to be related to a reduction in cellular damage induced by reactive oxygen species. There is increasing evidence that sirtuins play an essential role in the reduction of mitochondrial oxidative stress during caloric restriction. We speculate that cardiac sirtuins attenuate the accumulation of oxidative damage associated with age by modifying specific mitochondrial proteins posttranscriptionally. Therefore, the distinct role of each sirtuin in the heart subjected to caloric restriction should be clarified to translate sirtuin biology into clinical practice.

\section{Introduction}

Aging is characterized by a progressive deterioration in physiological functions and metabolic processes, leading to an increase in morbidity and mortality. Although many theories have been proposed to explain the aging process, neither of them appears to be fully satisfactory. Table 1 presents a summary of the major aging theories to date $[1,2]$. The free radical theory of aging originally stated that free radicals generated endogenously cause oxidative modification of cellular components; the accumulation of oxidative damage with aging results in cellular dysfunction and eventually cell death [3-5]. The free radical theory has been expanded in light of the fact that reactive oxygen species (ROS) are constantly produced in cells under normal condition and cells have a higher antioxidant capacity per se [3,5-7]. The oxidative stress theory of aging proposes that the formation of ROS including nonradical hydrogen peroxide and reactive nitrogen species, peroxynitrite, is the major generator of cellular damage and senescence. The age-related increase in oxidative damage to DNA, lipids, and proteins has been well documented $[3,5-7]$. The mitochondrion is the main source and target of ROS. Mitochondria have been a central focus of the aging theory because of their critical role in bioenergetics, oxidant production, and regulation of cell death [712]. According to the hypothesized role of mitochondria in the aging process, organs that exhibit high rate of oxygen consumption throughout an individual's lifetime, such as the heart, brain, and kidney, may be especially prone to oxidative damage [8]. However, ROS are not just unwanted byproducts of oxidative phosphorylation in the respiratory chain; they are also highly regulated signal molecules involved in the cellular stress response.

Numerous experimental interventions designed to regulate the aging process have been attempted heretofore. To date, an established intervention that has been consistently shown to slow the rate of aging and to increase both mean and maximal lifespan in various species is lifelong caloric restriction (CR) [11, 13, 14]. The beneficial effects of lifelong CR may derive, at least in part, from a reduction of oxidative 
TABLE 1: Major biological theories of aging.

(A) The programmed theory

(1) Programmed theory Telomere shortening theory

(2) Neuroendocrine theory

(3) Immunological theory
Aging is the result of a sequential switching of certain genes. Telomere plays a role in the genomic instability with aging.

Biological clocks act through the neurohumoral system to control the pace of aging.

The immune system is programmed to decline, which leads to an increased vulnerability to acute and chronic inflammation, resulting in aging and death.

(B) The damage or error theory (Nonprogrammed theory)

(1) Wear and tear theory

(2) Rate of living theory Metabolic theory

(3) Cross-linking theory Glycation theory

(4) Free radical theory Oxidative stress theory Mitochondrial theory

(5) Somatic DNA damage theory
Cells and tissues have vital parts that wear out, that leads to aging.

The greater a rate of basal oxygen metabolism, the shorter its lifespan.

The accumulation of modified constituents, such as cross-linked and glycated proteins, damages cells and tissues, resulting in aging.

Free radicals and reactive oxygen species (ROS) cause cellular damage and the accumulation of oxidative damage leads to aging. Mitochondria are a main source of ROS and also a target of ROS.

DNA damages occur continuously in living cells. Most of these damages are repaired, whereas some accumulate, resulting in cellular dysfunction and aging. In particular, damages to mitochondrial DNA lead to mitochondrial dysfunction. damage in organs and tissues [8, 11-15]. In contrast, although overexpression of antioxidant enzymes and antioxidant supplement diets have had some degree of success in attenuating age-associated physiological dysfunction and extending mean lifespan, they have not extended maximal lifespan [16-19]. Clinical investigations demonstrate that antioxidant treatment has either no effect or detrimental effects on health beneficial outcomes in cancer, diabetes, cardiovascular disease, and overall mortality $[20,21]$. These results suggest that modest ROS production promotes longevity by inducing the innate adaptive response against oxidative stress; this mechanism may be essential for the development of overall stress resistance and lifespan extension $[19,22]$. A favorable response to a low dose of poison is called hormesis. By extension, the beneficial response of mitochondria-derived ROS is named mitohormesis [22]. Since there are contradictory reports on the impact of exogenous antioxidant treatment on mitochondrial biogenesis and endogenous antioxidant defense, experiments that are more definitive are needed to address this issue.

In this paper, we discuss how cardiac mitochondrial dysfunction and oxidative stress contribute to cardiac aging and how CR regulates them.

\section{Mitochondrial Theory of Aging}

ROS are produced cellularly by enzymatic and nonenzymatic sources. Any electron-transferring protein and/or enzymatic system produce ROS as byproducts of electron transfer reactions. Although ROS are produced from $\mathrm{NADPH}$ oxidase, cyclooxygenases, peroxisomes, xanthine oxidase, cytochrome $P$-450, and others, mitochondria appear to produce the majority of oxidants [7, 8, 10-12, 23]. During essential oxygen-dependent ATP production in the electron transport chain (ETC), ROS is generated as a product of electron leakage from complex I and complex III where oxygen is reduced to form the superoxide radical (Figure 1) [23]. The generation of ROS in mitochondria is reported to account for $\sim 1-2 \%$ of total oxygen consumption under reducing conditions [24]. However, the intramitochondrial concentrations of superoxide are maintained at very low steady-state levels by mitochondrial superoxide dismutase (SOD), which is present at very high concentrations [25].

In addition to being a main source of ROS, mitochondria are a target for oxidative damage. ROS derived from mitochondrial respiration attack mitochondrial constituents. In particular, the accumulation of somatic mutations in mitochondrial DNA (mtDNA) by oxidative stress is believed to play a key role in physiological decline associated with aging [6-8, 10-12, 26]. MtDNA is located at the mitochondrial matrix where ROS are actively generated [7, 26]. Furthermore, mtDNA lacks protective histones and has relatively low DNA repair capacity $[7,26]$. ROS-induced mtDNA damage causes mtDNA mutations if mtDNA damage is not promptly repaired $[16,27]$. There is a positive relationship between the increase in oxidative damage to mtDNA and the ageassociated increase in mtDNA deletions and point mutations [2]. Because mtDNA encodes 13 of mitochondrial proteins in the ETC complexes $[28,29]$, mtDNA mutations alter the coupling of electron transport and ATP production. Finally, mtDNA mutations increase electron leakage from the ETC complexes and further damage mtDNA, as well as other important organelles [9]. Therefore, the mitochondrion is believed to be the key organelle in the cellular aging process.

\section{Age-Associated Alterations in Cardiac Mitochondrial Function}

The heart exhibits a highly aerobic metabolism due to the abundance of large mitochondria, which produce the huge amount of ATP for continuous contraction. Therefore, 


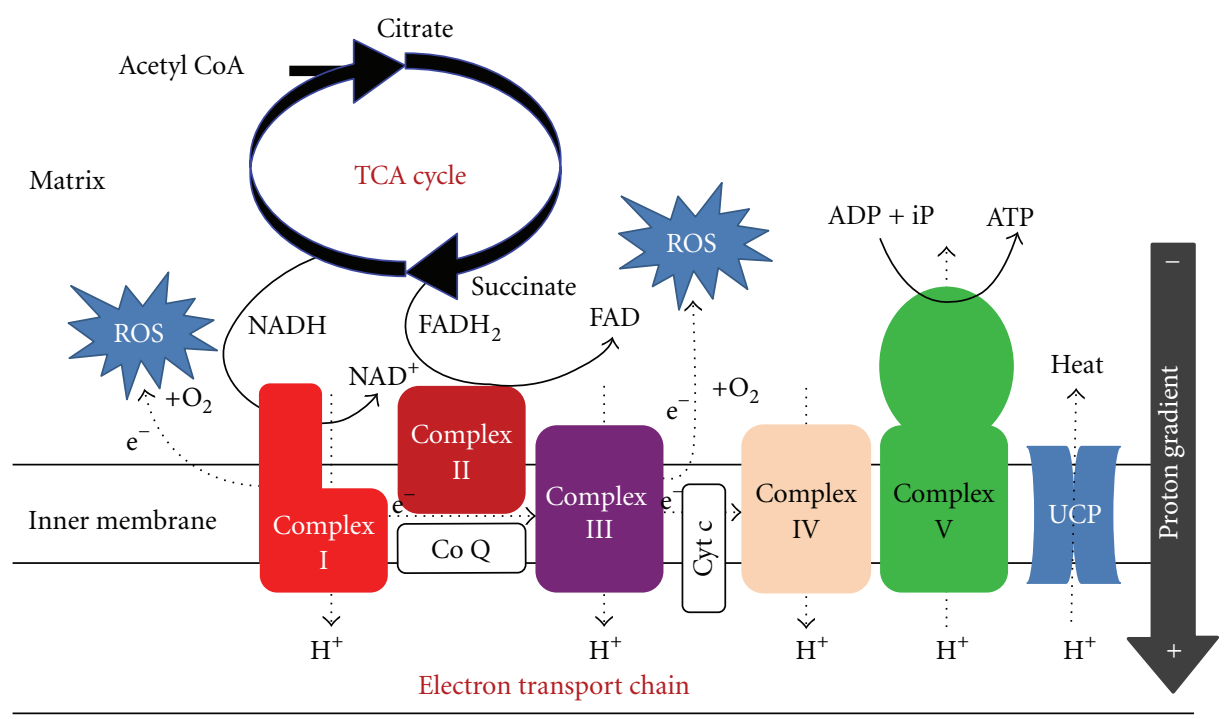

Outer membrane

Cytosol

FIGURE 1: The electron transport chain (ETC) responsible for ATP and ROS production in mitochondria. ROS: reactive oxygen species, TCA: tricarboxylic acid, NADH \& $\mathrm{NAD}^{+}$: nicotinamide adenine dinucleotide reduced form \& oxidized form, FADH2 \& FAD; flavin adenine dinucleotide reduced form and oxidized form, ADP: adenosine diphosphate, ATP: adenosine triphosphate, Co Q: coenzyme Q, Cyt c: cytochrome c, UCP: uncoupling protein.

the relationship between aging and mitochondrial bioenergetics in cardiomyocytes has been an important research area for many years. However, the interpretation of ageassociated alterations in cardiac mitochondrial function has been complicated by several factors. The heart contains two structurally similar but biochemically distinct mitochondrial populations $[8,30,31]$. Subsarcolemmal mitochondria are located beneath the plasma membrane, whereas interfibrillar mitochondria are arranged in parallel with myofibrils $[8,30$, 31]. Although evidence demonstrates that 2 populations of mitochondria differ in morphology and function, the isolation procedure to evaluate age-associated alterations in cardiac mitochondrial function yields either subsarcolemmal mitochondria alone or a mixed population of both mitochondria in most reports [31-34]. In addition, cardiomyocytes with dysfunctional mitochondria might be likely to drop out via apoptosis, necrosis, and/or autophagy thus further complicating the detection of bioenergetic changes in cardiac mitochondria with aging because only relatively healthy mitochondria are obtained after the isolation procedure [8]. Compared with those observed in cardiac diseases, alterations in cardiac mitochondria observed in aging might occur heterogeneously in the whole heart and at very low level among mitochondria. Therefore, the early feature of cardiac mitochondrial alterations might be masked by individual differences.

Whether mitochondrial oxidative phosphorylation is gradually impaired with aging remains controversial $[33,35-$ 38]. Some studies have demonstrated a decline in oxygen consumption with age $[36,38]$, but others have reported no change $[35,37]$. Fannin et al. reported that interfibrillar mitochondria but not subsarcolemmal mitochondria obtained from aged rat hearts (24 and 28 months old) exhibited less protein production and oxidative phosphorylation rates, compared with those from adult rat heart (6 months old) [32]. This finding might explain the inconsistency in ageassociated alterations in oxidative phosphorylation: the ratio of interfibrillar mitochondria to total mitochondria preparations would be expected to vary.

Whether the activity of each ETC complex decreases with aging is also undetermined [39-46]. Among the five ETC complexes, complex I appears to be susceptible to age-associated decline in activity in the mammalian heart $[39,40,42$, 44-46], although some studies demonstrated no significant change in complex I activity with age [41, 43]. Complex I is believed to be a main source of ROS derived from mitochondrial ETC and to be responsible for the increase in mitochondrial ROS production with aging [12, 23, 42, 44, 47]. Because 7 of 13 mtDNA-encoded polypeptides in the ETC are found in complex I $[28,29]$, this complex is likely to be most commonly affected by aging, if the mitochondrial theory of aging proves to be true [42]. In addition, the activities of complexes III and IV, which also contain mtDNAencoded proteins, have been reported to decrease with aging $[32,34,39-41,45]$. Complex III is another source of ROS production in the mitochondrial ETC $[12,23,34]$. In contrast, most investigations have shown that the activity of complex II appears to be unaffected or rather enhanced by aging $[40,41,43,45,46]$. Since mtDNA does not encode any of the polypeptides in complex II [48], this finding further 
supports the mitochondrial theory of aging. In addition, protein levels in complexes III, IV, and V were reported to decrease in hearts obtained from old monkeys [38]. Gómez et al. demonstrated that formation of supercomplexes consisting of complexes I, III, and IV decreased in the aged rat heart [49]. Some investigators reported that a decrease in mitochondrial cardiolipin, which is located in the inner mitochondrial membrane and bound to cytochrome c, is closely associated with the decrease in the activity of complex III $[50,51]$. However, others have reported that aging does not alter mitochondrial cardiolipin content or composition in subsarcolemmal or interfibrillar mitochondria isolated from rat hearts [44]. Complex V activity is also reported to decline with aging in the heart [52-54]; oxidative modification of $\beta$ polypeptides in the F1 complex of complex V may be responsible, at least in part, for this phenomenon [54]. Other types of protein modification, such as 3-nitrotyrosine, have also been found in each ETC complex [55]. Recently, an agerelated decline in complexes I and V activity that correlated with increased oxidative modification has been reported in the aged mouse heart, although there was no change in the protein expression levels of them [56]. They may contribute to the decline in mitochondrial function associated with aging.

The mitochondrial proteome has been comprehensively analyzed to clarify the effect of aging and CR on mitochondrial proteins. Chang et al. demonstrated that the effect of aging on the mitochondrial proteome in the heart appears to be slighter than that in the liver, and CR has a minor effect on these changes [57]. These results strongly suggest that posttranslational modifications of mitochondrial proteins are more important than transcriptional changes in the development of age-associated alterations in mitochondrial function and the effect of CR [58].

In conclusion, mounting evidence supports an age-associated decline in cardiac mitochondrial function, especially in the activity of complexes I, III, and IV. However, future studies are required to determine the exact mechanism by which aging impairs cardiac mitochondrial function and to further characterize differential effects of age on the subsarcolemmal and interfibrillar mitochondria populations in the heart.

\section{Mitochondrial Oxidative Damage in the Aged Heart}

As a major source of ROS production, mitochondria themselves are susceptible to oxidative damage. In fact, the accumulation of oxidative damage in mitochondria is observed in various organs of aged animals $[5,10,11,13,59,60]$. ROS produced by mitochondria damages mitochondrial and nuclear DNA, lipids, and proteins. Oxidized DNA may mutate, lipid peroxidation can attenuate integrity of cellular and intracellular membrane, and oxidized proteins lose their enzymatic activity $[16,60,61]$. These events negatively affect mitochondrial and cellular function and contribute to the decline in physiological function with age. Although oxidative damage increases with age in nuclear DNA and mtDNA [27], Barja and Herrero demonstrated that levels of 8oxo-7,8-dihydro- $2^{\prime}$-deoxyguanosine (8-oxodG), an indicator of oxidative DNA damage, were 4-fold higher in mtDNA compared with levels in nuclear DNA in hearts obtained from 8 mammalian species with various lifespans [59]. They found an inverse correlation between 8-oxodG levels in mtDNA and maximal lifespan among the different species. Since the rate of repair for 8-oxodG is believed to be similar between nuclear DNA and mtDNA [62], the higher levels of 8-oxodG in mtDNA are likely caused, at least in part, by the chronic exposure of mtDNA to ROS due to its location in mitochondria. The repair activity for damaged mtDNA increases in the aged heart, indirectly supporting the idea that the rate of mtDNA damage increases with age in the heart [63].

Protein and lipid constituents in mitochondria are also susceptible to oxidative modifications. Increasing evidence demonstrates that polyunsaturated fatty acids contained in membrane lipids are vulnerable to peroxidation by ROS, and lipid peroxidation has been shown to increase in cardiac mitochondria with aging $[33,35,64,65]$. Lipid peroxidation is a major contributor to the age-associated loss of membrane fluidity; two aldehyde lipid peroxidation products, malondialdehyde (MDA) and 4-hydroxy-2-nonenal (HNE), are primarily responsible for this phenomenon [61]. MDA and HNE rapidly react with proteins and exhibit various cytotoxic effects. In particular, HNE is highly reactive with other molecules and exerts numerous effects including inhibition of protein and DNA synthase, enzymatic inactivation, and subsequent cellular dysfunction [66-68]. HNE-modified proteins become resistant to proteolytic degradation and act as noncompetitive inhibitors of the proteasome [67]. In mitochondria, HNE is mainly detoxified by aldehyde dehydrogenase (ALDH) under physiological conditions [66]. Transgenic mice carrying an Aldh2 gene with a single nucleotide polymorphism (Aldh2*2), which impairs ALDH activity, exhibit a senescent phenotype at an early stage of life [69]. However, whether the enzymatic activity and/or the expression levels of ALDH are affected in the aged heart has not been evaluated. Measurement of protein carbonyl content is a commonly used method for assessing protein oxidation. Protein carbonyls can be formed via several mechanisms including site-specific metal-catalyzed oxidation of lysine, arginine, proline, and threonine residues; glycation reactions; and interaction of amino acid side chains with lipid peroxidation products such as MDA and $\operatorname{HNE}[60,70]$. The accumulation of oxidized proteins may play a role in the loss of physiological function with age because oxidized proteins lose catalytic activity and are prone to forming large and potentially cytotoxic protein aggregates $[5,60,70]$. Several studies have shown an age-associated increase in protein carbonyls in cardiac mitochondria and concluded that this increase may contribute, at least in part, to the decline in mitochondrial function with aging $[33,35,39,71]$.

\section{The Effect of CR on Cardiac Oxidative Stress}

CR is the only experimental intervention that has consistently shown to slow the rate of aging and increase both mean and maximal lifespan in various species [11, 13-15]. The exact 
TABLE 2: Seven members of sirtuin family.

\begin{tabular}{|c|c|c|c|c|}
\hline Sirtuin & Class & $\begin{array}{c}\text { Cellular } \\
\text { localization }\end{array}$ & Enzymatic activity & Target molecules \\
\hline SIRT1 & I & $\begin{array}{l}\text { Nucleus, Cytosol, } \\
\text { (Mitochondria) }\end{array}$ & Deacetylase & $\begin{array}{l}\text { Histone } \mathrm{H} 3, \mathrm{H} 4 \text {, p53, nuclear factor } \kappa \mathrm{B}(\mathrm{NF} \kappa \mathrm{B}) \text {, peroxisome } \\
\text { proliferator-activated receptor- } \gamma \text { co-activator } 1 \alpha \text { (PGCl } \alpha) \text {, forkhead box } \mathrm{O} \\
\text { transcriptional factors (FoxO) } 1 \& 3 \text {, Notch, hypoxia-inducible factor } 1 \alpha \\
(\mathrm{HIFl} \alpha) \text {, liver X receptor (LXR), farnesoid X receptor (FXR), } \\
\text { sterol-response element-binding protein 1c (SREBP1c), p300, endothelial } \\
\text { nitric oxide synthase (eNOS), peroxisome proliferator-activated receptor } \\
\text { (PPAR) } \gamma \text {, CREB-regulated transcription co-activator } 2 \text { (CRTC2), and so } \\
\text { forth }\end{array}$ \\
\hline SIRT2 & I & $\begin{array}{l}\text { Cytosol, } \\
\text { (Nucleus) }\end{array}$ & Deacetylase & $\begin{array}{l}\alpha \text {-tubulin, phosphoenolpyruvate carboxykinase (PEPCK), FoxO1, } \\
\text { partitioning defective } 3 \text { homologue (PAR3) }\end{array}$ \\
\hline SIRT3 & I & Mitochondria & Deacetylase & $\begin{array}{l}\text { Long-chain acyl CoA dehydrogenase (LCAD), 3-hydroxy-3-methylglutaryl } \\
\text { CoA synthase } 2 \text { (HMGCS2), glutamate dehydrogenase (GDH), NADH } \\
\text { dehydrogenase ubiquinone } 1 \text { subcomplex } 9 \text { (NDUFA9), superoxide } \\
\text { dismutase } 2 \text { (SOD2), isocitrate dehydrogenase } 2 \text { (IDH2), cyclophilin D } \\
\text { (CypD), acetyl-CoA synthetase 2 (AceCS2), LKB1, and so forth }\end{array}$ \\
\hline SIRT4 & II & Mitochondria & ADP-ribosyltransferase & GDH \\
\hline SIRT5 & III & Mitochondria & $\begin{array}{c}\text { Deacetylase } \\
\text { Demalonylase } \\
\text { Desuccinylase }\end{array}$ & Carbamoyl phosphate synthetase 1 (CPS1) \\
\hline SIRT6 & IV & Nucleus & $\begin{array}{l}\text { Deacetylase } \\
\text { ADP-ribosyltransferase }\end{array}$ & Histone H3K9, H3K56 \\
\hline SIRT7 & IV & Nucleolus & Unknown & Histone H3K18, p53, RNA polymerase I \\
\hline
\end{tabular}

mechanisms by which CR extends lifespan have not fully been evaluated, but mounting evidence demonstrates that a reduction in oxidative stress contributes, at least in part, to the antiaging effects of CR $[11,13-15,19,71,72]$. CR attenuates the age-associated increase in mitochondrial ROS production, lipid peroxidation, protein oxidation, and oxidative damage of mtDNA in various organs.

Increasing evidence demonstrates that $\mathrm{CR}$ has pleiotropic effects on the cardiovascular system $[15,73]$. Several studies have reported that CR significantly decreases oxidative damage in the aged heart $[47,71,74-76]$. The levels of 8 oxodG were lower in cardiac mitochondria obtained from CR rats than in those from ad libitum-fed controls $[47,76]$. Numerous reports indicate that CR reduces oxidative damage to proteins and lipids in cardiac mitochondria. However, the exact mechanisms involved remain to be determined. In other words, how CR attenuates cardiac oxidative stress has not been established.

Recently, sirtuin 3 (SIRT3) located in mitochondria was shown to play an essential role in enhancing antioxidant defense during CR in the liver and the brain [77, 78]. SIRT3 is a member of the sirtuin family which comprises seven proteins (SIRT1-SIRT7), whose tissue specificity, subcellular localization, enzymatic activity, and target proteins vary (Table 2) [79-81]. Sirtuins have received significant attention since the discovery that a yeast sirtuin, silent information regulator (Sir) 2, extends yeast lifespan. Sir2 was identified as an $\mathrm{NAD}^{+}$-dependent histone deacetylase. Sirtuins deacetylate histones and a wide range of transcriptional regulators and intracellular molecules, thereby controlling their activity. Although it remains controversial whether sirtuins mediate lifespan extension afforded by CR, sirtuins do regulate various aspects of the $\mathrm{CR}$ response, namely, glucose homeostasis, insulin secretion, fat metabolism, stress resistance, and physical activity. However, direct evidence that sirtuins play a key role in the reduction of oxidative stress in the cardiovascular system was lacking.

Several studies have reported that CR decreases mitochondrial ROS production in the heart, but failed to clarify the mechanism underlying this observation [47, 82-84]. Figure 2 presents possible mechanisms by which CR attenuates cardiac oxidative damage, which are verified in the latter half of this paper.

\section{Antioxidant Defense in the CR Heart}

CR might modify mitochondrial ROS production via enhanced mitochondrial biogenesis associated with amplified antioxidant mechanisms. Nisoli et al. demonstrated that 3 months of CR enhanced mitochondrial biogenesis in the murine heart and in skeletal muscle [85]. They concluded that activation of SIRT1 contributes to an increase in mitochondrial biogenesis in the CR heart by upregulating gene involved in mitochondrial biogenesis, including nuclear respiratory factor 1 and peroxisome proliferator-activated receptor $\gamma$ coactivator 1. Activation of SIRT1 might enhance the expression of manganese SOD (MnSOD) by activating Forkhead box protein $\mathrm{O} 1$ as observed in cardiomyopathy hamsters treated with resveratrol [86]. However, we did not find any increase in the mitochondrial DNA and protein content 


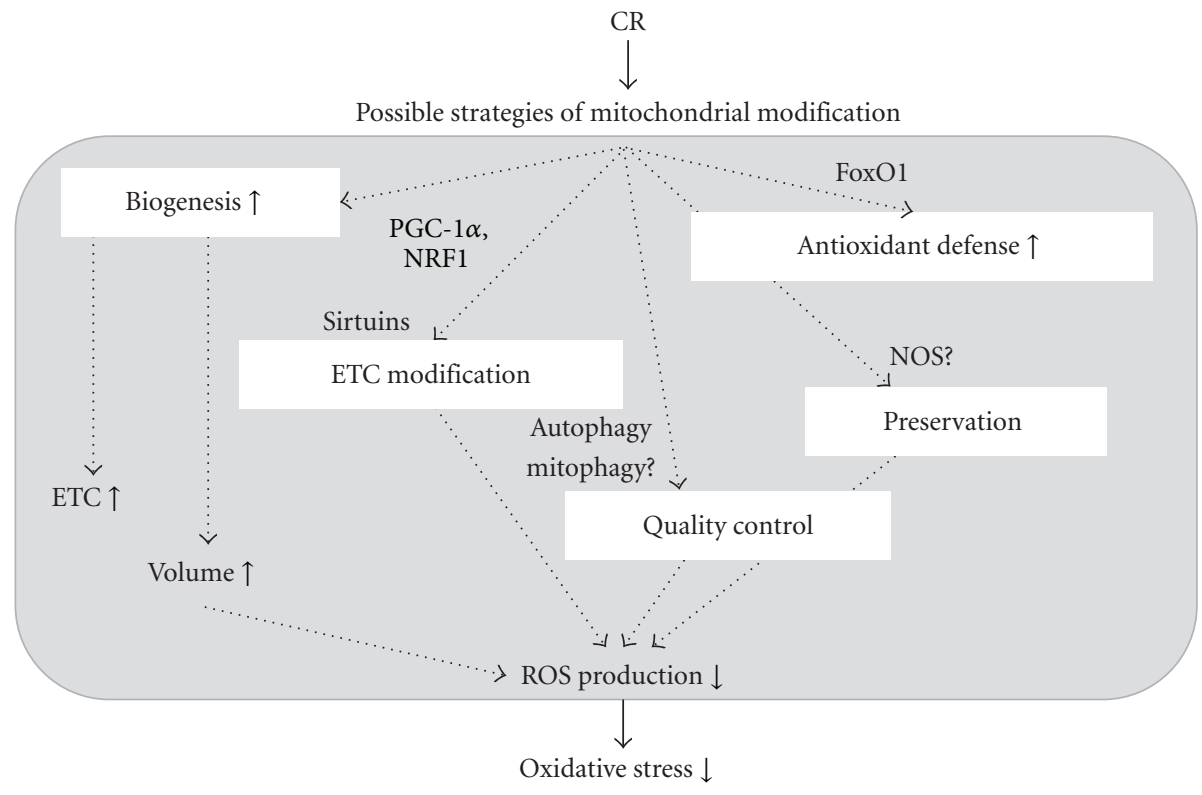

FIgURE 2: Possible mechanisms by which CR attenuates oxidative stress. CR: caloric restriction, PGC-1 $\alpha$ : peroxisome proliferator-activated receptor $\gamma$ coactivator $1 \alpha$, NRF1: nuclear respiratory factor 1, FoxO1: forkhead transcriptional factor 1, NOS: nitric oxide synthase.

or in the expression of MnSOD in middle-aged rat hearts treated with 6 months of $\mathrm{CR}$, indicating that $\mathrm{CR}$ did not enhance mitochondrial biogenesis associated with amplified antioxidant mechanisms [84]. In this regard, Colom et al. failed to find any increase in the expression levels of MnSOD in 18-month-old rat hearts treated with 3 months of CR [82]. More recently, SIRT3 was reported to enhance the enzymatic activity of MnSOD by direct deacetylation [77]. However, it has not been determined yet whether MnSOD is the target of SIRT3 in cardiomyocytes. Judge et al. demonstrated that the activity of MnSOD was significantly reduced in subsarcolemmal mitochondria from young rat hearts treated with CR for 8 weeks, associated with a decrease in $\mathrm{H}_{2} \mathrm{O}_{2}$ production [83]. Therefore, the simplest explanation is that CR attenuates mitochondrial oxidative damage by suppressing mitochondrial ROS production, rather than by enhancing antioxidant defense.

\section{Mitochondrial Function in the CR Heart}

Results on the effect of CR on basal mitochondrial function in the heart are inconsistent $[47,74,75,82,84,87]$. We have evaluated the enzymatic activity of the ETC, baseline mitochondrial respiration, and mitochondrial $\mathrm{H}_{2} \mathrm{O}_{2}$ production in the hearts of middle-aged (12-month-old) rats treated with 6 months of CR and those of middle-aged rats fed AL [84]. The only difference was that $\mathrm{CR}$ attenuated maximal $\mathrm{H}_{2} \mathrm{O}_{2}$ production in mitochondria; this was assessed by adding rotenone (complex I inhibitor) in the presence of pyruvate/ malate.

Several studies demonstrated that prolonged CR improved mitochondrial function at baseline in aged hearts, but other studies failed to find any changes in mitochondria [58]. The discrepancy among studies seems to depend on the strain of rats used, the age of rats analyzed, and the duration of CR treatment. Niemann et al. demonstrated that 6 months of CR improved mitochondrial respiration and enhanced the enzymatic activity of complex I in 30-monthold rat hearts but not in 12-month-old rat hearts [75]. Our results were consistent with their findings in middle-aged rat hearts, suggesting that $\mathrm{CR}$ has little effect on basal mitochondrial function in the middle-aged heart, probably because mitochondrial function is maintained at a high level [84].

We speculated that the difference in mitochondrial function between $\mathrm{AL}$ and $\mathrm{CR}$ might be more remarkable under stressful conditions, such as ischemia/reperfusion. As expected, CR preserved state 3 respiration and increased the respiratory control index in the presence of pyruvate/malate in the ischemic-reperfused heart [84]. These findings suggest that the mitochondria in the CR heart are well coupled during the ischemia/reperfusion sequence. Mitochondria obtained from ischemic-reperfused CR hearts produced less $\mathrm{H}_{2} \mathrm{O}_{2}$ in the presence of pyruvate/malate [84], suggesting that mitochondria in the CR heart produce less ROS during early reperfusion. The preservation of mitochondrial respiration with attenuated $\mathrm{H}_{2} \mathrm{O}_{2}$ production in the $\mathrm{CR}$ heart subjected to ischemia/reperfusion strongly suggests that mitochondria in the CR heart are more resistant to ischemia/reperfusion. Suppressing mitochondrial ROS production under various stresses could attenuate the long-term accumulation of oxidative damage and might retard cardiac senescence in the CR heart [58].

\section{Possible Mechanism of Attenuated Oxidative Damage in the CR Heart}

Increasing evidence indicates that several enzymes target mitochondria under stress. Thus, it is likely that mitochondrial proteins are modified posttranscriptionally and 
regulated to allow the cell to adapt to various stresses. Among many forms of protein modification, we investigated the acetylation/deacetylation of mitochondrial proteins in order to clarify the effects of CR. Three members of the sirtuin family, SIRT3, SIRT4, and SIRT5, localize specifically in mitochondria and play an important role in regulating mitochondrial metabolism and function by modifying target mitochondrial proteins in various organs under metabolic stress [79-81]. In addition, we previously demonstrated that cardioprotection afforded by prolonged CR is associated with an increase in SIRT1 in the nuclear fraction [88].

We found that the levels of proteins acetylated at lysine residues increased with age in the mitochondrial fraction; furthermore, these changes were partially attenuated with CR [84]. There was no increase in the expression of SIRT3, 4, or 5 in mitochondrial fractions obtained from the CR heart. However, CR significantly increased $\mathrm{NAD}^{+}$-dependent deacetylase activity in total heart homogenate and the mitochondrial fraction [84]. These results suggest that CR enhances the activity of sirtuins and that activated sirtuins might be responsible for the reverse effect of $\mathrm{CR}$ on mitochondrial protein acetylation associated with aging.

Among the mitochondrial proteins deacetylated during $\mathrm{CR}$, we focused on the following 2 mitochondrial proteins involved in the ETC: NADH-ubiquinone oxidoreductase $75 \mathrm{kDa}$ subunit (NDUFS1) and cytochrome bcl complex Rieske subunit [84]. NDUFS1 is the largest subunit of complex I and is a component of the iron-sulfur fragment of the enzyme. It faces the mitochondrial matrix, receives electrons from $\mathrm{NADH}$, and passes them to the downstream iron-sulfur protein clusters. The Rieske subunit is also a component of the iron-sulfur fragment of cytochrome bcl complex and transfers electrons to cytochrome c. Although the exact mechanism has not been clarified, we speculate that deacetylation of these proteins might stabilize electron transfer through the ETC and reduce electron leakage during ischemia/reperfusion sequence. As described previously, many mitochondrial proteins in the ETC are targets for oxidative modifications under various stresses. In contrast, it is plausible that some types of posttranscriptional modification play a protective role against oxidative modification under stress [58].

\section{Reduction in Mitochondrial Burden during $\mathbf{C R}$}

Among various substrates that the heart can utilize, free fatty acids are believed to be the major substrate for the adult heart under physiological conditions. In contrast, utilization of glucose in the heart predominantly increases under pathophysiological conditions such as ischemia/reperfusion and pressure overload. The efficiency of ATP production per one molecule of substrate is better when fatty acids are utilized, but mitochondria require high levels of oxygen for $\beta$ oxidation during fatty acid metabolism. Finally, both glucosederived and fatty acid-derived acetyl CoA enter the TCA cycle. Therefore, the question arises whether the preference of energy substrate is altered in the CR heart.
Sung et al. evaluated the effect of short-term CR (for 5 weeks) on myocardial metabolism [89]. They found that there were no differences in rates of palmitate oxidation and glycolysis during aerobic perfusion between the CR and the AL hearts. However, glucose oxidation was increased by $175 \%$ in the CR heart, compared to the AL heart. Consistent with this finding, glucose-derived acetyl CoA production in the TCA cycle was increased in the CR heart. A high rate of glucose oxidation in the CR heart might contribute to improve myocardial energetics under stressful conditions. However, there is no data regarding the effect of long-term $\mathrm{CR}$ on myocardial metabolism and this issue remains to be clarified.

Experimental studies demonstrate that animals subjected to $\mathrm{CR}$ exhibit a decrease in systemic blood pressure and heart rate, compared with controls fed AL [90, 91]. A clinical observation in which individuals who had been on CR diet for an average of 6 years were compared with age-matched healthy individuals on typical American diet indicated a change in blood pressure similar to that observed in animal experiments [92]. Thus, the decrease in cardiovascular burden during CR might contribute, at least in part, to the antiaging effect of CR on cardiovascular senescence. Recent studies demonstrated that SIRT1 and endothelial nitric oxide synthase (eNOS) colocalize in endothelial cells and that SIRT1 deacetylates eNOS, stimulating eNOS activity, and increasing NO production in endothelial cells [15, 93, 94]. Thus, it is plausible that CR activates SIRT1 and improves NO bioavailability in the cardiovascular system, resulting in a decrease in blood pressure during CR [95]. Reduction of mitochondrial work in the heart by pharmacological intervention has potential for mimicking the effect of longterm CR. In this regard, either pharmacological inhibition of renin-angiotensin system throughout the lifespan or genetic disruption of the angiotensin type 1 receptor gene, which led to a decrease in blood pressure, was reported to promote longevity and retard cardiovascular senescence in rodents $[96,97]$.

\section{Implications for Human Aging and Age-Associated Cardiovascular Diseases}

Although it is well accepted that oxidative stress is involved in the aging process and the antiaging effect of CR is closely related to a reduction in ROS-induced cellular damage, there is very little data that demonstrates these relationships in humans or lager animals. Therefore, the translatability to human aging for short-lived small species is always a debating issue. A clear distinction should be drawn between the observation that CR appears to work in humans and a recommendation that individuals embark on its practice. A lot of issues have remained to be understood about the effect of CR on humans.

An increased baseline level of oxidative damage to DNA is reported to be associated with age [98] and several age-related diseases including cardiovascular diseases [99]. Higher levels of protein carbonyls are observed with increased age in healthy human subjects [100]. In contrast, a recent investigation from comprehensive assessment of the long-term 
effect of reducing intake of energy (CALERIE) demonstrated that DNA damage was reduced from baseline after 6 months in individuals assigned to CR, but not in controls [101]. Although the effects of $\mathrm{CR}$ on lifespan in nonhuman primates were inconsistent $[102,103]$, preferable outcomes on ageassociated oxidative damage by CR could be observed in nonhuman primates [104]. These results suggest that the oxidative stress theory of aging would apply to humans, at least in part.

Clearly, the use of CR mimetics is much easier to incorporate into clinical practice than lifelong CR. The role of sirtuins on lifespan extension by CR remains controversial, but there is the fact that sirtuins regulate various aspects of the CR response. In addition, SIRT1 plays an important role in cardiac adaptive response to various stresses such as ischemia/reperfusion [105] and activation of SIRT1 confers antioxidative and anti-inflammatory effects in the vasculature, resulting in attenuated vascular senescence [106]. Thus, activators of sirtuins are potentially useful for managing ageassociated cardiovascular diseases. Our finding further suggests targeted modification of specific mitochondrial proteins by relevant sirtuin-activating compounds is a promising approach for controlling cardiovascular senescence. We found that low-dose resveratrol mimics the effect of CR on deacetylation of specific mitochondrial proteins belonging to the ETC [84]. Resveratrol is reported to be a potentially cardioprotective compound, but not a specific sirtuin activator $[107,108]$. Thus, future studies should focus on discovering other sirtuin-activating compounds that deacetylate specific mitochondrial proteins with high specificity and efficiency. The distinct role of sirtuin member should be clarified to understand the mechanism by which CR modifies mitochondrial bioenergetics and cellular oxidative stress in the cardiovascular system. At the present time, the easy use of sirtuin-activating compounds in clinical settings should be avoided.

\section{Conflict of Interests}

The author declares that he has no conflict of interests.

\section{Acknowledgments}

This work was supported by a Grant from the Ministry of Education, Culture, and Science, Japan (2010-2012) and by a Grant from the Vehicle Racing Commemorative Foundation (2012).

\section{References}

[1] K. Jin, "Modern biological theories of aging," Aging and Disease, vol. 1, no. 2, pp. 72-74, 2010.

[2] B. R. Troen, “The biology of aging," Mount Sinai Journal of Medicine, vol. 70, no. 1, pp. 3-22, 2003.

[3] K. B. Beckman and B. N. Ames, "The free radical theory of aging matures," Physiological Reviews, vol. 78, no. 2, pp. 547-581, 1998.

[4] D. Harman, "Aging: a theory based on free radical and radiation chemistry," Journal of Gerontology, vol. 11, no. 3, pp. 298-300, 1956.
[5] R. S. Sohal, "Role of oxidative stress and protein oxidation in the aging process," Free Radical Biology and Medicine, vol. 33, no. 1, pp. 37-44, 2002.

[6] A. W. Linnane, S. Marzuki, T. Ozawa, and M. Tanaka, "Mitochondrial DNA mutations as an important contributor to ageing and degenerative diseases," Lancet, vol. 1, no. 8639, pp. 642645, 1989.

[7] J. Miquel, A. C. Economos, J. Fleming, and J. E. Johnson, "Mitochondrial role in cell aging," Experimental Gerontology, vol. 15, no. 6, pp. 575-591, 1980.

[8] S. Judge and C. Leeuwenburgh, "Cardiac mitochondrial bioenergetics, oxidative stress, and aging," American Journal of Physiology, vol. 292, no. 6, pp. C1983-C1992, 2007.

[9] G. Lenaz, C. Bovina, M. D’Aurelio et al., "Role of mitochondria in oxidative stress and aging," Annals of the New York Academy of Sciences, vol. 959, pp. 199-213, 2002.

[10] G. López-Lluch, P. M. Irusta, P. Navas, and R. de Cabo, "Mitochondrial biogenesis and healthy aging," Experimental Gerontology, vol. 43, no. 9, pp. 813-819, 2008.

[11] B. J. Merry, "Oxidative stress and mitochondrial function with aging - the effects of calorie restriction," Aging Cell, vol. 3, no. 1, pp. 7-12, 2004.

[12] M. H. Vendelbo and K. S. Nair, "Mitochondrial longevity pathways," Biochimica et Biophysica Acta, vol. 1813, no. 4, pp. 634-644, 2011.

[13] E. J. Masoro, "Overview of caloric restriction and ageing," Mechanisms of Ageing and Development, vol. 126, no. 9, pp. 913922, 2005.

[14] R. S. Sohal and R. Weindruch, "Oxidative stress, caloric restriction, and aging," Science, vol. 273, no. 5271, pp. 59-63, 1996.

[15] Z. Ungvari, C. Parrado-Fernandez, A. Csiszar, and R. De Cabo, "Mechanisms underlying caloric restriction and lifespan regulation: implications for vascular aging," Circulation Research, vol. 102, no. 5, pp. 519-528, 2008.

[16] T. M. Hagen, R. Moreau, J. H. Suh, and F. Visioli, "Mitochondrial decay in the aging rat heart: evidence for improvement by dietary supplementation with acetyl-L-carnitine and/or lipoic acid," Annals of the New York Academy of Sciences, vol. 959, pp. 491-507, 2002.

[17] W. C. Orr and R. S. Sohal, "Extension of life-span by overexpression of superoxide dismutase and catalase in Drosophila melanogaster," Science, vol. 263, no. 5150, pp. 1128-1130, 1994.

[18] S. E. Schriner, N. J. Linford, G. M. Martin et al., "Medecine: extension of murine life span by overexpression of catalase targeted to mitochondria," Science, vol. 308, no. 5730, pp. 19091911, 2005.

[19] T. J. Schulz, K. Zarse, A. Voigt, N. Urban, M. Birringer, and M. Ristow, "Glucose restriction extends Caenorhabditis elegans life span by inducing mitochondrial respiration and increasing oxidative stress," Cell Metabolism, vol. 6, no. 4, pp. 280-293, 2007.

[20] G. Bjelakovic, D. Nikolova, L. L. Gluud, R. G. Simonetti, and C. Gluud, "Antioxidant supplements for prevention of mortality in healthy participants and patients with various diseases," Cochrane Database of Systematic Reviews, no. 2, article CD007176, 2008.

[21] D. P. Vivekananthan, M. S. Penn, S. K. Sapp, A. Hsu, and E. J. Topol, "Use of antioxidant vitamins for the prevention of cardiovascular disease: meta-analysis of randomised trials," Lancet, vol. 361, no. 9374, pp. 2017-2023, 2003. 
[22] M. Ristow and K. Zarse, "How increased oxidative stress promotes longevity and metabolic health: the concept of mitochondrial hormesis (mitohormesis)," Experimental Gerontology, vol. 45, no. 6, pp. 410-418, 2010.

[23] D. C. Wallace, "A mitochondrial paradigm of metabolic and degenerative diseases, aging, and cancer: a dawn for evolutionary medicine," Annual Review of Genetics, vol. 39, pp. 359-407, 2005.

[24] A. Boveris and B. Chance, "The mitochondrial generation of hydrogen peroxide. General properties and effect of hyperbaric oxygen," Biochemical Journal, vol. 134, no. 3, pp. 707-716, 1973.

[25] S. K. Dhar and D. K. St Clair, "Manganese superoxide dismutase regulation and cancer," Free Radical Biology and Medicine, vol. 52, no. 11-12, pp. 2209-2222, 2012.

[26] M. K. Shigenaga, T. M. Hagen, and B. N. Ames, "Oxidative damage and mitochondrial decay in aging," Proceedings of the National Academy of Sciences of the United States of America, vol. 91, no. 23, pp. 10771-10778, 1994.

[27] M. L. Hamilton, H. Van Remmen, J. A. Drake et al., "Does oxidative damage to DNA increase with age?" Proceedings of the National Academy of Sciences of the United States of America, vol. 98, no. 18, pp. 10469-10474, 2001.

[28] S. Anderson, A. T. Bankier, and B. G. Barrell, "Sequence and organization of the human mitochondrial genome," Nature, vol. 290, no. 5806, pp. 457-465, 1981.

[29] M. J. Bibb, R. A. Van Etten, C. T. Wright, M. W. Walberg, and D. A. Clayton, "Sequence and gene organization of mouse mitochondrial DNA," Cell, vol. 26, no. 2, part 2, pp. 167-180, 1981.

[30] J. W. Palmer, B. Tandler, and C. L. Hoppel, "Biochemical properties of subsarcolemmal and interfibrillar mitochondria isolated from rat cardiac muscle," Journal of Biological Chemistry, vol. 252, no. 23, pp. 8731-8739, 1977.

[31] J. W. Palmer, B. Tandler, and C. L. Hoppel, "Biochemical differences between subsarcolemmal and interfibrillar mitochondria from rat cardiac muscle: effects of procedural manipulations," Archives of Biochemistry and Biophysics, vol. 236, no. 2, pp. 691702, 1985.

[32] S. W. Fannin, E. J. Lesnefsky, T. J. Slabe, M. O. Hassan, and C. L. Hoppel, "Aging selectively decreases oxidative capacity in rat heart interfibrillar mitochondria," Archives of Biochemistry and Biophysics, vol. 372, no. 2, pp. 399-407, 1999.

[33] S. Judge, M. J. Young, A. Smith, T. Hagen, and C. Leeuwenburgh, "Age-associated increases in oxidative stress and antioxidant enzyme activities in cardiac interfibrillar mitochondria: implications for the mitochondrial theory of aging," The FASEB Journal, vol. 19, no. 3, pp. 419-421, 2005.

[34] E. J. Lesnefsky, T. I. Gudz, S. Moghaddas et al., "Aging decreases electron transport complex III activity in heart interfibrillar mitochondria by alteration of the cytochrome c binding site," Journal of Molecular and Cellular Cardiology, vol. 33, no. 1, pp. 37-47, 2001.

[35] T. Cocco, P. Sgobbo, M. Clemente et al., "Tissue-specific changes of mitochondrial functions in aged rats: effect of a long-term dietary treatment with N-acetylcysteine," Free Radical Biology and Medicine, vol. 38, no. 6, pp. 796-805, 2005.

[36] E. Delaval, M. Perichon, and B. Friguet, "Age-related impairment of mitochondrial matrix aconitase and ATP-stimulated protease in rat liver and heart," European Journal of Biochemistry, vol. 271, no. 22, pp. 4559-4564, 2004.
[37] M. S. Manzelmann and H. J. Harmon, "Lack of age-dependent changes in rat heart mitochondria," Mechanisms of Ageing and Development, vol. 39, no. 3, pp. 281-288, 1987.

[38] L. Yan, H. Ge, H. Li et al., "Gender-specific proteomic alterations in glycolytic and mitochondrial pathways in aging monkey hearts," Journal of Molecular and Cellular Cardiology, vol. 37, no. 5, pp. 921-929, 2004.

[39] A. L. Andreu, M. A. Arbos, A. Perez-Martos et al., "Reduced mitochondrial DNA transcription in senescent rat heart," Biochemical and Biophysical Research Communications, vol. 252, no. 3, pp. 577-581, 1998.

[40] C. Castelluccio, A. Baracca, R. Fato et al., "Mitochondrial activities of rat heart during ageing," Mechanisms of Ageing and Development, vol. 76, no. 2-3, pp. 73-88, 1994.

[41] L. K. Kwong and R. S. Sohal, "Age-related changes in activities of mitochondrial electron transport complexes in various tissues of the mouse," Archives of Biochemistry and Biophysics, vol. 373, no. 1, pp. 16-22, 2000.

[42] G. Lenaz, C. Bovina, C. Castelluccio et al., "Mitochondrial complex I defects in aging," Molecular and Cellular Biochemistry, vol. 174, no. 1-2, pp. 329-333, 1997.

[43] Ò. Miró, J. Casademont, E. Casals et al., "Aging is associated with increased lipid peroxidation in human hearts, but not with mitochondrial respiratory chain enzyme defects," Cardiovascular Research, vol. 47, no. 3, pp. 624-631, 2000.

[44] G. Petrosillo, M. Matera, N. Moro, F. M. Ruggiero, and G. Paradies, "Mitochondrial complex I dysfunction in rat heart with aging: critical role of reactive oxygen species and cardiolipin," Free Radical Biology and Medicine, vol. 46, no. 1, pp. 8894, 2009.

[45] S. Sugiyama, M. Takasawa, M. Hayakawa, and T. Ozawa, "Changes in skeletal muscle, heart and liver mitochondrial electron transport activities in rats and dogs of various ages," Biochemistry and Molecular Biology International, vol. 30, no. 5, pp. 937-944, 1993.

[46] M. Takasawa, M. Hayakawa, S. Sugiyama, K. Hattori, T. Ito, and T. Ozawa, "Age-associated damage in mitochondrial function in rat hearts," Experimental Gerontology, vol. 28, no. 3, pp. 269280, 1993.

[47] R. Gredilla, A. Sanz, M. Lopez-Torres, and G. Barja, "Caloric restriction decreases mitochondrial free radical generation at complex I and lowers oxidative damage to mitochondrial DNA in the rat heart," The FASEB Journal, vol. 15, no. 9, pp. 1589-1591, 2001.

[48] T. Ozawa, "Genetic and functional changes in mitochondria associated with aging," Physiological Reviews, vol. 77, no. 2, pp. 425-464, 1997.

[49] L. A. Gómez, J. S. Monette, J. D. Chavez, C. S. Maier, and T. M. Hagen, "Supercomplexes of the mitochondrial electron transport chain decline in the aging rat heart," Archives of Biochemistry and Biophysics, vol. 490, no. 1, pp. 30-35, 2009.

[50] E. J. Lesnefsky, P. Minkler, and C. L. Hoppel, "Enhanced modification of cardiolipin during ischemia in the aged heart," Journal of Molecular and Cellular Cardiology, vol. 46, no. 6, pp. 1008-1015, 2009.

[51] G. Paradies, F. M. Ruggiero, G. Petrosillo, and E. Quagliariello, "Peroxidative damage to cardiac mitochondria: cytochrome oxidase and cardiolipin alterations," FEBS Letters, vol. 424, no. 3, pp. 155-158, 1998.

[52] S. M. K. Davies, A. Poljak, M. W. Duncan, G. A. Smythe, and M. P. Murphy, "Measurements of protein carbonyls, ortho- and 
meta-tyrosine and oxidative phosphorylation complex activity in mitochondria from young and old rats," Free Radical Biology and Medicine, vol. 31, no. 2, pp. 181-190, 2001.

[53] E. Guerrieri, G. Capozza, A. Fratello, F. Zanotti, and S. Papa, "Functional and molecular changes in F0F1 ATP-synthase of cardiac muscle during aging," Cardioscience, vol. 4, no. 2, pp. 93-98, 1993.

[54] C. S. Yarian, D. Toroser, and R. S. Sohal, "Aconitase is the main functional target of aging in the citric acid cycle of kidney mitochondria from mice," Mechanisms of Ageing and Development, vol. 127, no. 1, pp. 79-84, 2006.

[55] J. Kanski, A. Behring, J. Pelling, and C. Schöneich, "Proteomic identification of 3-nitrotyrosine-containing rat cardiac proteins: effects of biological aging," American Journal of Physiology, vol. 288, no. 1, pp. H371-H381, 2005.

[56] K. B. Choksi, J. E. Nuss, J. H. DeFord, and J. Papaconstantinou, "Age-related alterations in oxidatively damaged proteins of mouse skeletal muscle mitochondrial electron transport chain complexes," Free Radical Biology and Medicine, vol. 45, no. 6, pp. 826-838, 2008.

[57] J. Chang, J. E. Cornell, H. Van Remmen, K. Hakala, W. F. Ward, and A. Richardson, "Effect of aging and caloric restriction on the mitochondrial proteome," Journals of Gerontology A, vol. 62, no. 3, pp. 223-234, 2007.

[58] K. Shinmura, "Post-translational modification of mitochondrial proteins by caloric restriction: possible involvement in caloric restriction-induced cardioprotection," Trends in Cardiovascular Medicine, vol. 23, no. 1, pp. 18-25, 2013.

[59] G. Barja and A. Herrero, "Oxidative damage to mitochondrial DNA is inversely related to maximum life span in the heart and brain of mammals," The FASEB Journal, vol. 14, no. 2, pp. 312$318,2000$.

[60] E. R. Stadtman, "Protein oxidation in aging and age-related diseases," Annals of the New York Academy of Sciences, vol. 928, pp. 22-38, 2001.

[61] J. J. Chen and B. P. Yu, "Alterations in mitochondrial membrane fluidity by lipid peroxidation products," Free Radical Biology and Medicine, vol. 17, no. 5, pp. 411-418, 1994.

[62] R. M. Anson, D. L. Croteau, R. H. Stierum, C. Filburn, R. Parsell, and V. A. Bohr, "Homogenous repair of singlet oxygen-induced DNA damage in differentially transcribed regions and strands of human mitochondrial DNA," Nucleic Acids Research, vol. 26, no. 2, pp. 662-668, 1998.

[63] N. C. Souza-Pinto, D. L. Croteau, E. K. Hudson, R. G. Hansford, and V. A. Bohr, "Age-associated increase in 8-oxo-deoxyguanosine glycosylase/AP lyase activity in rat mitochondria," Nucleic Acids Research, vol. 27, no. 8, pp. 1935-1942, 1999.

[64] L. L. Ji, D. Dillon, and E. Wu, "Myocardial aging: antioxidant enzyme systems and related biochemical properties," American Journal of Physiology, vol. 261, no. 2, pp. R386-R392, 1991.

[65] S. Phaneuf and C. Leeuwenburgh, "Cytochrome c release from mitochondria in the aging heart: a possible mechanism for apoptosis with age," American Journal of Physiology, vol. 282, no. 2, pp. R423-R430, 2002.

[66] G. R. Budas, M. H. Disatnik, and D. Mochly-Rosen, "Aldehyde dehydrogenase 2 in cardiac protection: a new therapeutic target?" Trends in Cardiovascular Medicine, vol. 19, no. 5, pp. 158$164,2009$.

[67] B. Friguet, E. R. Stadtman, and L. I. Szweda, "Modification of glucose-6-phosphate dehydrogenase by 4-hydroxy-2-nonenal.
Formation of cross-linked protein that inhibits the multicatalytic protease," Journal of Biological Chemistry, vol. 269, no. 34, pp. 21639-21643, 1994.

[68] K. Okada, C. Wangpoengtrakul, T. Osawa, S. Toyokuni, K. Tanaka, and K. Uchida, "4-Hydroxy-2-nonenal-mediated impairment of intracellular proteolysis during oxidative stress. Identification of proteasomes as target molecules," Journal of Biological Chemistry, vol. 274, no. 34, pp. 23787-23793, 1999.

[69] J. Endo, M. Sano, T. Katayama et al., "Metabolic remodeling induced by mitochondrial aldehyde stress stimulates tolerance to oxidative stress in the heart," Circulation Research, vol. 105, no. 11, pp. 1118-1127, 2009.

[70] S. Adams, P. Green, R. Claxton et al., "Reactive carbonyl formation by oxidative and non-oxidative pathways," Front Biosci, vol. 6, pp. A17-A24, 2001.

[71] K. Shinmura, K. Tamaki, M. Sano et al., "Impact of longterm caloric restriction on cardiac senescence: caloric restriction ameliorates cardiac diastolic dysfunction associated with aging," Journal of Molecular and Cellular Cardiology, vol. 50, no. 1, pp. 117-127, 2011.

[72] R. Pamplona, M. Portero-Otín, J. Requena, R. Gredilla, and G. Barja, "Oxidative, glycoxidative and lipoxidative damage to rat heart mitochondrial proteins is lower after 4 months of caloric restriction than in age-matched controls," Mechanisms of Ageing and Development, vol. 123, no. 11, pp. 1437-1446, 2002.

[73] K. Shinmura, "Cardiovascular protection afforded by caloric restriction: essential role of nitric oxide synthase," Geriatrics and Gerontology International, vol. 11, no. 2, pp. 143-156, 2011.

[74] A. J. Lambert, B. Wang, J. Yardley, J. Edwards, and B. J. Merry, "The effect of aging and caloric restriction on mitochondrial protein density and oxygen consumption," Experimental Gerontology, vol. 39, no. 3, pp. 289-295, 2004.

[75] B. Niemann, Y. Chen, H. Issa, R. E. Silber, and S. Rohrbach, "Caloric restriction delays cardiac ageing in rats: role of mitochondria," Cardiovascular Research, vol. 88, no. 2, pp. 267-276, 2010.

[76] A. Sanz, R. Gredilla, R. Pamplona et al., "Effect of insulin and growth hormone on rat heart and liver oxidative stress in control and caloric restricted animals," Biogerontology, vol. 6, no. 1, pp. 15-26, 2005.

[77] X. Qiu, K. Brown, M. D. Hirschey, E. Verdin, and D. Chen, "Calorie restriction reduces oxidative stress by SIRT3-mediated SOD2 activation," Cell Metabolism, vol. 12, no. 6, pp. 662-667, 2010.

[78] S. Someya, W. Yu, W. C. Hallows et al., "Sirt3 mediates reduction of oxidative damage and prevention of age-related hearing loss under caloric restriction," Cell, vol. 143, no. 5, pp. 802-812, 2010.

[79] M. Abdellatif, "Sirtuins and pyridine nucleotides," Circulation Research, vol. 111, no. 5, pp. 642-656, 2012.

[80] R. H. Houtkooper, E. Pirinen, and J. Auwerx, "Sirtuins as regulators of metabolism and healthspan," Nature Reviews Molecular Cell Biology, vol. 13, no. 4, pp. 225-238, 2012.

[81] T. Nakagawa and L. Guarente, "Sirtuins at a glance," Journal of Cell Science, vol. 124, no. 6, pp. 833-838, 2011.

[82] B. Colom, J. Oliver, P. Roca, and F. J. Garcia-Palmer, "Caloric restriction and gender modulate cardiac muscle mitochondrial $\mathrm{H}_{2} \mathrm{O}_{2}$ production and oxidative damage," Cardiovascular Research, vol. 74, no. 3, pp. 456-465, 2007.

[83] S. Judge, A. Judge, T. Grune, and C. Leeuwenburgh, "Shortterm CR decreases cardiac mitochondrial oxidant production but increases carbonyl content," American Journal of Physiology, vol. 286, no. 2, pp. R254-R259, 2004. 
[84] K. Shinmura, K. Tamaki, M. Sano et al., "Caloric restriction primes mitochondria for ischemic stress by deacetylating specific mitochondrial proteins of the electron transport chain," Circulation Research, vol. 109, no. 4, pp. 396-406, 2011.

[85] E. Nisoli, C. Tonello, A. Cardile et al., "Cell biology: calorie restriction promotes mitochondrial biogenesis by inducing the expression of eNOS," Science, vol. 310, no. 5746, pp. 314-317, 2005.

[86] M. Tanno, A. Kuno, T. Yano et al., "Induction of manganese superoxide dismutase by nuclear translocation and activation of SIRT1 promotes cell survival in chronic heart failure," Journal of Biological Chemistry, vol. 285, no. 11, pp. 8375-8382, 2010.

[87] B. Drew, P. A. Dirks, C. Selman et al., "Effects of aging and caloric restriction on mitochondrial energy production in gastrocnemius muscle and heart," American Journal of Physiology, vol. 284, no. 2, pp. R474-R480, 2003.

[88] K. Shinmura, K. Tamaki, and R. Bolli, "Impact of 6-mo caloric restriction on myocardial ischemic tolerance: possible involvement of nitric oxide-dependent increase in nuclear Sirt1," American Journal of Physiology, vol. 295, no. 6, pp. H2348-H2355, 2008.

[89] M. M. Sung, C. L. Soltys, G. Masson, J. J. Boisvenue, and J. R. Dyck, "Improved cardiac metabolism and activation of the RISK pathway contributes to improved post-ischemic recovery in calorie restricted mice," Journal of Molecular Medicine, vol. 89, no. 3, pp. 291-302, 2011.

[90] L. M. Hunt, E. W. Hogeland, M. K. Henry, and S. J. Swoap, "Hypotension and bradycardia during caloric restriction in mice are independent of salt balance and do not require ANP receptor," American Journal of Physiology, vol. 287, no. 4, pp. H1446-H1451, 2004.

[91] J. B. Young, D. Mullen, and L. Landsberg, "Caloric restriction lowers blood pressure in the spontaneously hypertensive rat," Metabolism, vol. 27, no. 12, pp. 1711-1714, 1978.

[92] L. Fontana, T. E. Meyer, S. Klein, and J. O. Holloszy, "Longterm calorie restriction is highly effective in reducing the risk for atherosclerosis in humans," Proceedings of the National Academy of Sciences of the United States of America, vol. 101, no. 17, pp. 6659-6663, 2004.

[93] I. Mattagajasingh, C. S. Kim, A. Naqvi et al., "SIRT1 promotes endothelium-dependent vascular relaxation by activating endothelial nitric oxide synthase," Proceedings of the National Academy of Sciences of the United States of America, vol. 104, no. 37, pp. 14855-14860, 2007.

[94] H. Ota, M. Akishita, M. Eto, K. Iijima, M. Kaneki, and Y. Ouchi, "Sirtl modulates premature senescence-like phenotype in human endothelial cells," Journal of Molecular and Cellular Cardiology, vol. 43, no. 5, pp. 571-579, 2007.

[95] A. E. Civitarese, S. Carling, L. K. Heilbronn et al., "Calorie restriction increases muscle mitochondrial biogenesis in healthy humans," PLoS Medicine, vol. 4, no. 3, article e76, pp. 485-494, 2007.

[96] N. Basso, R. Cini, A. Pietrelli, L. Ferder, N. A. Terragno, and F. Inserra, "Protective effect of long-term angiotensin II inhibition," American Journal of Physiology, vol. 293, no. 3, pp. H1351H1358, 2007.

[97] A. Benigni, D. Corna, C. Zoja et al., "Disruption of the Ang II type 1 receptor promotes longevity in mice," Journal of Clinical Investigation, vol. 119, no. 3, pp. 524-530, 2009.

[98] H. Massudi, R. Grant, N. Braidy, J. Guest, B. Farnsworth, and G. J. Guillemin, "Age-associated changes in oxidative stress and
$\mathrm{NAD}^{+}$metabolism in human tissue," PLoS One, vol. 7, no. 7, Article ID e42357, 2012.

[99] A. R. Collins, C. M. Gedik, B. Olmedilla, S. Southon, and M. Bellizzi, "Oxidative DNA damage measured in human lymphocytes: large differences between sexes and between countries, and correlations with heart disease mortality rates," The FASEB Journal, vol. 12, no. 13, pp. 1397-1400, 1998.

[100] P. Voss and W. Siems, "Clinical oxidation parameters of aging," Free Radical Research, vol. 40, no. 12, pp. 1339-1349, 2006.

[101] L. K. Heilbronn, L. De Jonge, M. I. Frisard et al., "Effect of 6month calorie restriction on biomarkers of longevity, metabolic adaptation, and oxidative stress in overweight individuals: a randomized controlled trial," Journal of the American Medical Association, vol. 295, no. 13, pp. 1539-1548, 2006.

[102] R. J. Colman, R. M. Anderson, S. C. Johnson et al., "Caloric restriction delays disease onset and mortality in rhesus monkeys," Science, vol. 325, no. 5937, pp. 201-204, 2009.

[103] J. A. Mattison, G. S. Roth, T. Mark Beasley et al., "Impact of caloric restriction on health and survival in rhesus monkeys from the NIA study," Nature, vol. 489, no. 7415, pp. 318-321, 2012.

[104] T. A. Zainal, T. D. Oberley, D. B. Allison, L. I. Szweda, and R. Weindruch, "Caloric restriction of rhesus monkeys lowers oxidative damage in skeletal muscle," The FASEB Journal, vol. 14, no. 12, pp. 1825-1836, 2000.

[105] T. Yamamoto and J. Sadoshima, "Protection of the heart against ischemia/reperfusion by silent information regulator 1," Trends in Cardiovascular Medicine, vol. 21, no. 1, pp. 27-32, 2011.

[106] H. Ota, M. Eto, S. Ogawa, K. Iijima, M. Akishita, and Y. Ouchi, "Sirtl/eNOS axis as a potential target against vascular senescence, dysfunction and atherosclerosis," Journal of Atherosclerosis and Thrombosis, vol. 17, no. 5, pp. 431-435, 2010.

[107] V. W. Dolinsky and J. R. Dyck, "Calorie restriction and resveratrol in cardiovascular health and disease," Biochim Biophys Acta, vol. 1812, no. 11, pp. 1477-1489, 2011.

[108] N. L. Price, A. P. Gomes, A. J. Y. Ling et al., "SIRT1 is required for AMPK activation and the beneficial effects of resveratrol on mitochondrial function," Cell Metabolism, vol. 15, no. 5, pp. 675690, 2012. 


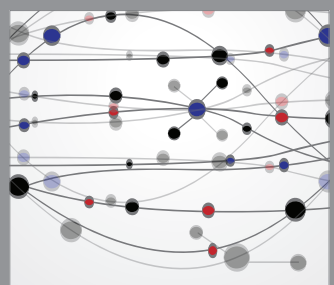

The Scientific World Journal
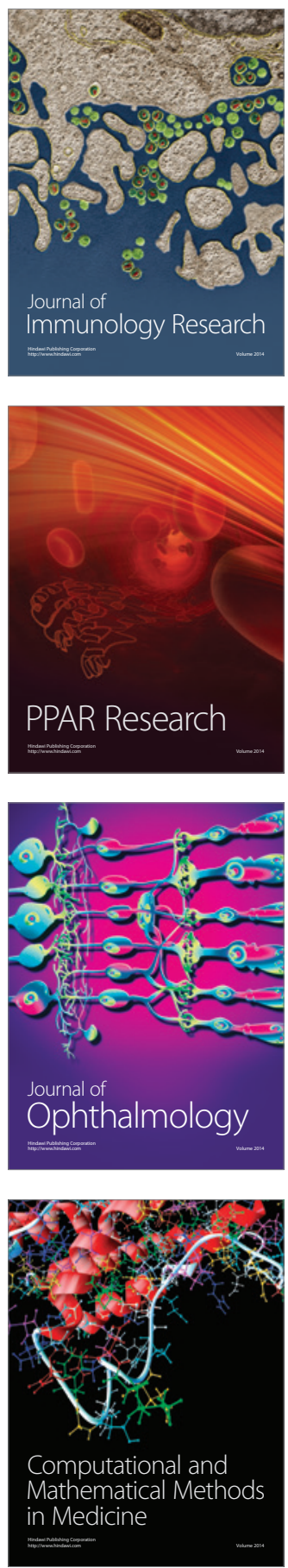

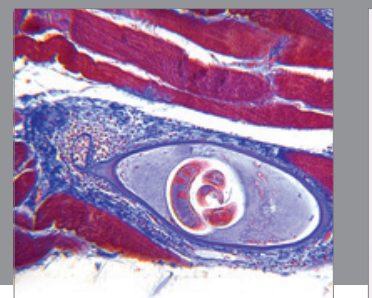

Gastroenterology

Research and Practice
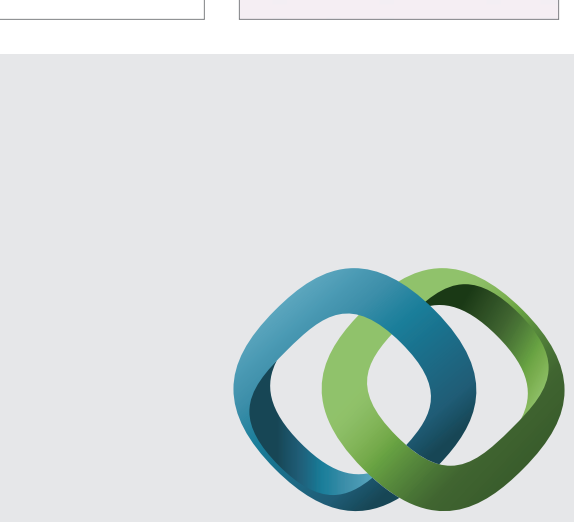

\section{Hindawi}

Submit your manuscripts at

http://www.hindawi.com
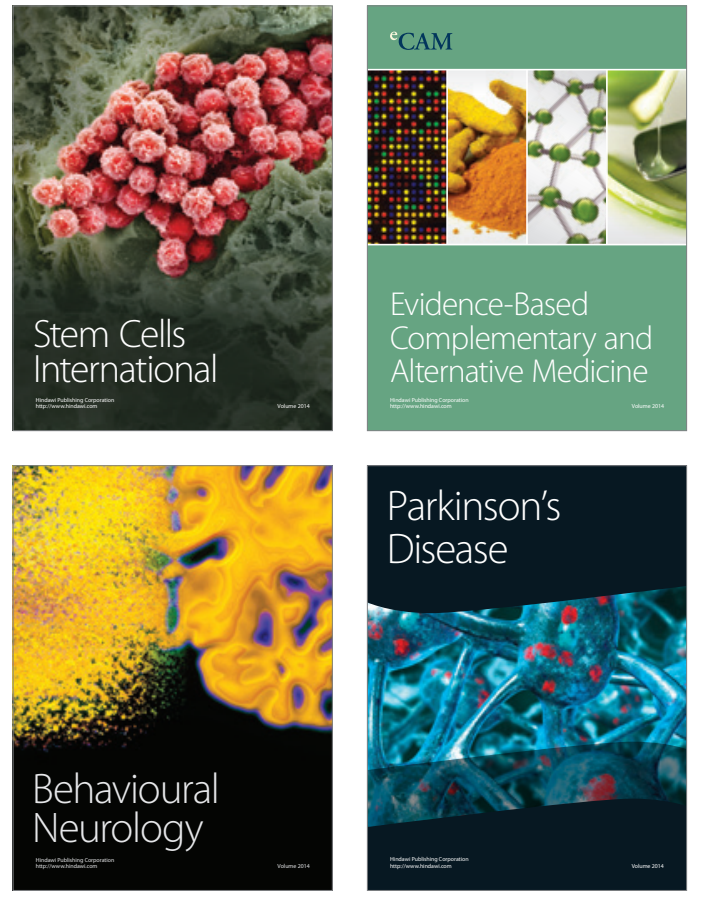
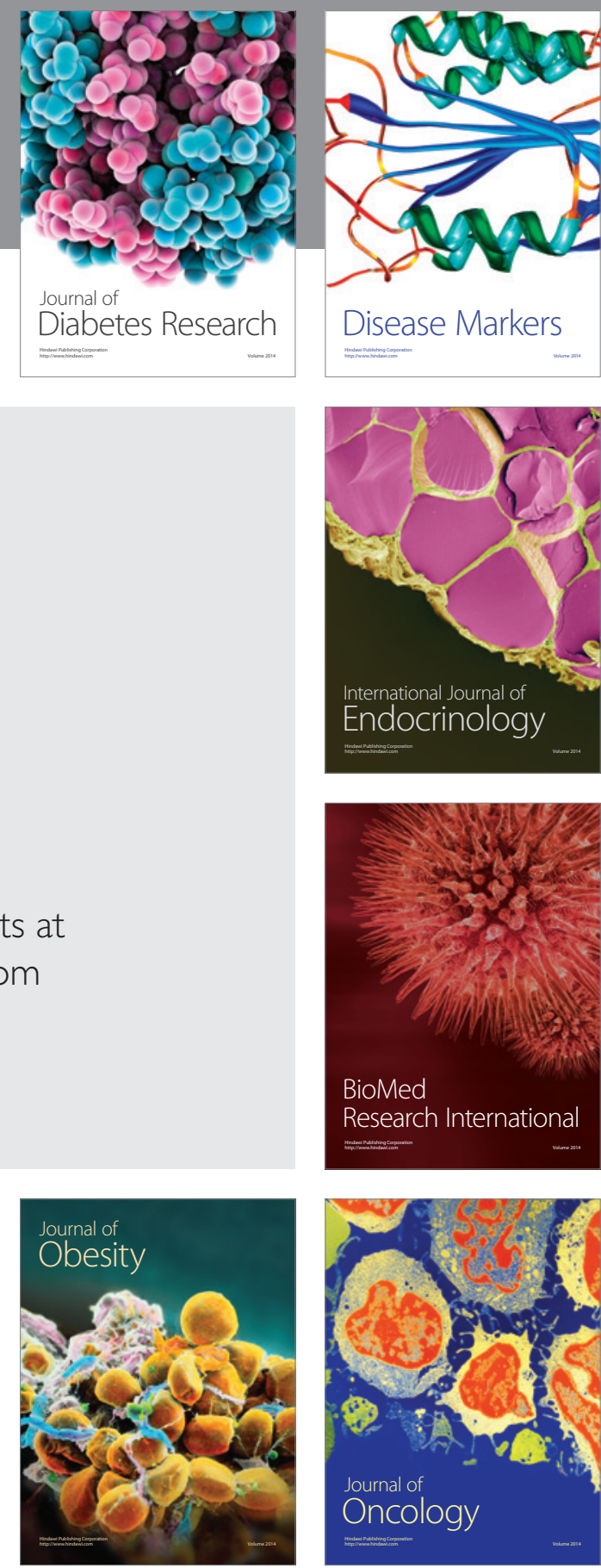

Disease Markers
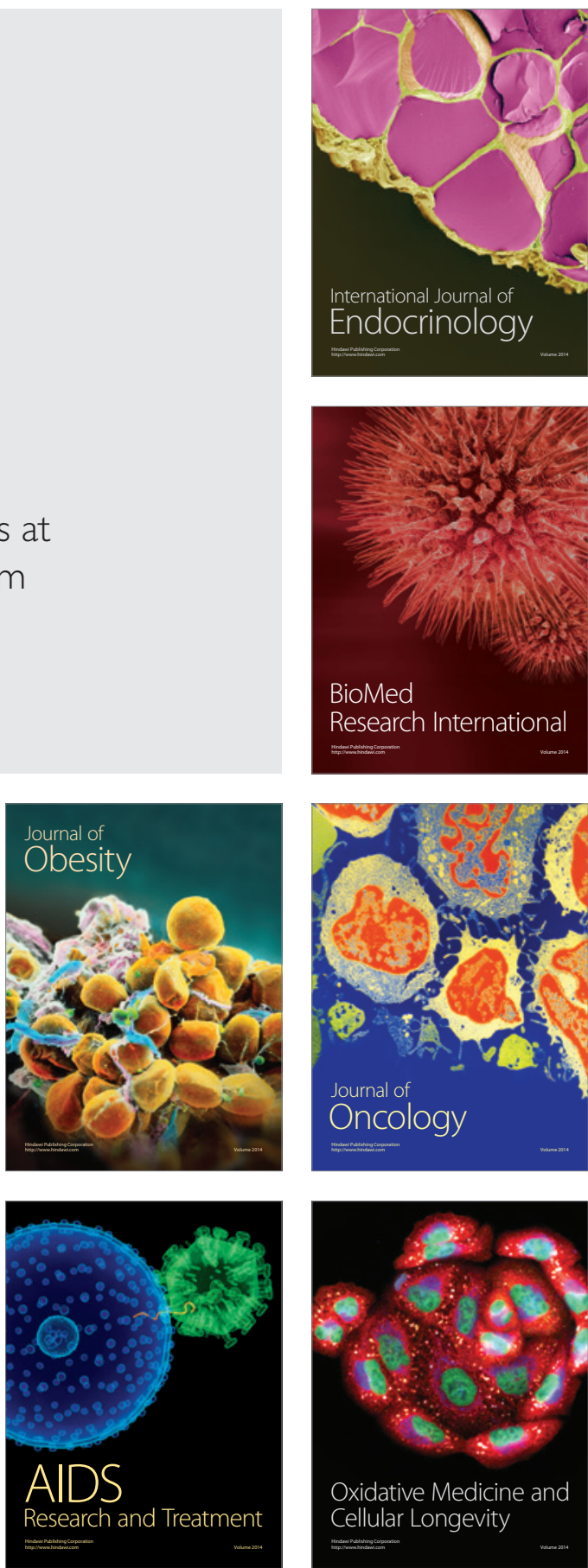